\title{
Efficient practices in railway ballast maintenance and quality assessment using GPR
}

\author{
L. Bianchini Ciampoli, A. Calvi \& A. Benedetto \\ Roma Tre University, Rome, Italy
}

F. Tosti \& A.M. Alani

University of West London, London, United Kingdom

\begin{abstract}
The need for effective and efficient railway maintenance is always more demanded all over the world as the main consequence of aging and degradation of infrastructures. Primarily, the filling of air voids within a railway ballast track-bed by fine-grained materials, coming up from the subballast layers by vibrations and capillarity effects, can heavily affect both the bearing and the draining capacity of the infrastructure with major impacts on safety. This occurrence is typically referred to as "fouling". When ballast is fouled, especially by clay, its internal friction angle is undermined, with serious lowering of the strength properties and increase of deformation rates of the whole rail track-bed. Thereby, a detailed and up-to-date knowledge of the quality of the railway substructure is mandatory for scheduling proper maintenance, with the final goal of optimizing the productivity while keeping the safety at the highest standard. This paper aims at reviewing a set of maintenance methodologies, spanning from the traditional and most employed ones, up to the most innovative approaches available in the market, with a special focus on the Ground Penetrating Radar (GPR) non-destructive testing (NDT) technique. The breakthrough brought by the application of new processing approaches is also analyzed and a methodological framework is given on some of the most recent and effective maintenance practices.
\end{abstract}

\section{STATEMENT OF THE PROBLEM}

Freights, bulk goods and commuters travel every day between cities, terminals and production poles upon ballasted rail tracks, all around the world. As a result of society development and technological enhancement, the speed of trains is increasing, as well as the requested performance of the track-bed. On the other hand, to ensure safety of the transport and highest standards of productivity, the railway network needs to be effectively and timely maintained, due to expected increased deformation rates by the passing loads.

A railway track-bed can be broadly divided into superstructure and substructure (Figure 1). The former includes steel rails, fastening system and sleepers, whilst the latter is composed of granular layers laying upon the subgrade, namely, ballast and subballast. In more detail, ballast is a homogeneously hard-rock-derived graded material. Its main functions are to resist to the stresses imposed by passing loads and transmit the vertical forces, properly attenuated, to the subgrade. Thereby, ballast holds the primary roles of retaining the track in its correct position, absorbing the acoustic waves within the air-filled voids, and ensuring proper drainage of meteoric water (Benedetto et al., 2016a).

Differential track settlements are prevalently related to the substructure, whose mechanical response, is the major concern of designers and maintenance managers (Indraratna 2016). Indeed, whilst the superstructure components react to forces elastically with negligible deformations, the stress cycles imposed by the passing loads induce relevant deformation into the granular layers composing the substructure (Chrismer 1985). This cyclic loading determines a breakage of the sharp corner of ballast grains and a fragmentation of the weaker particles. Thereby, the grading of the substructure changes (Selig \& Waters 1994, Ebrahimi et al. 2012) with implications on the mechanical behaviour and drainage capacity of the material.

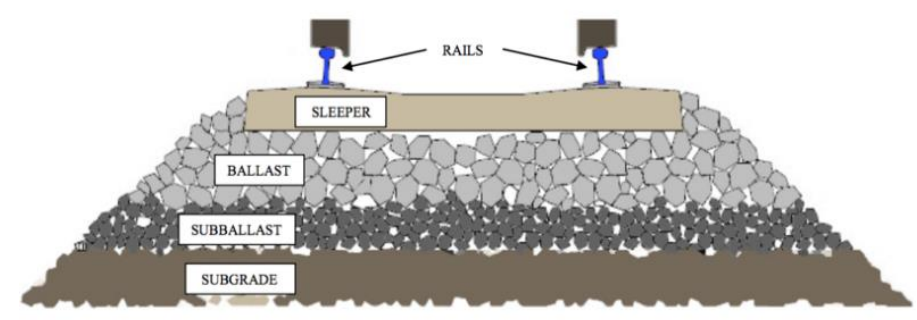

Figure 1. Typical railway track-bed cross-section.

Furthermore, the air-filled voids can get polluted by the deposit of concrete and steel dust produced at the ballast-sleeper and wheel-rail contact (Indraratna et al. 2014), respectively, by the pouring of coal dust from the passing freights (Huang et al. 2010, Tennakoon et al. 2015), and by the upward migration of fine clay particles from the subgrade along with capillary water (Al-Qadi et al., 2010). Mostly, regardless of the nature of the process, this filling of ballast voids by fine particles is referred to as 'fouling'. When the ballast is fouled, especially by clay, its internal friction angle is undermined, with serious lowering of the strength properties and increase of deformation rates of the whole rail track-bed. 
In view of the above, a detailed and up-to-date knowledge of the quality of the railway substructure is mandatory for scheduling proper maintenance, with the final goal of optimizing the productivity while keeping the safety at the highest standard.

This study evaluates a set of maintenance methodologies, spanning from traditional and most employed ones, up to the most innovative approaches available in the market, with a special mention to the Ground Penetrating Radar (GPR) non-destructive testing (NDT) technique, whose application in railways is increasingly attracting the attention of researchers, companies and administration. In Chapter 2, an overview of the up-to-date methodologies for maintaining railway ballast is given; hence, the GPR method, pros and cons are discussed in Section 3; then, in Section 4 , a scheme of efficient maintenance management is proposed; conclusions and research open issues are finally presented in Section 5.

\section{RAILWAY MAINTENANCE}

With regards to the aforementioned railway substructure, ballast plays a crucial role in maintaining serviceability of the track and ensuring its structural capacity (Al-Qadi et al. 2008, Al-Qadi et al. 2010). The fouling process causes degradation of the original substructure, and can compromise the performance of the railway track system. At worst, such an occurrence can increase the risk of derailment (De Chiara et al. 2014).

Thereby, monitoring and maintenance stages of track-beds are relevant to ensure high performance of the railway system over its service time, and granting the highest safety standards. At the same time, they are challenging tasks to be carried out due to the significant longitudinal extent of these infrastructures.

Traditional methods for the monitoring and assessment of ballast degradation are visual investigations, punctual drillings and diggings, which are carried out all along the track at discrete intervals. Nevertheless, these methods have several disadvantages. Indeed, visual inspections do not allow for subsurface investigation and understanding of the deep causes of the damage. On the other hand, drillings and diggings do not provide continuous data on the health conditions of the track, although they can return highly reliable information (Al-Qadi et al. 2008, Al-Qadi et al. 2010). Furthermore, these are labour intensive and time consuming (Shao et al. 2010).

Thereby, the recent focus is to move towards the use of time- and cost-effective technologies, capable of performing rapid and non-destructive inspection of the track-bed.

Amongst these technologies, two-dimensional (2D) (Cho et al. 2006) and three-dimensional (3D) (Sun et al. 2014) laser scanners, based on an optical method of investigation, are currently used for railway inspections. In more detail, 2D and 3D imaging methods have been used for assessing critical features such as size and shape of ballast particles.

In the last decades, GPR has attracted great interest in the assessment of railway ballast conditions. To that effect, there is a plenty of literature studies proving the capabilities of such a non-destructive tool to overcome the limits of the traditional investigation methodologies (Hyslip et al. 2003, Shao et al. 2010).

\section{GROUND PENETRATING RADAR}

\subsection{Working principles}

GPR is a geophysical inspection technique based on the transmission of electromagnetic (EM) waves towards a medium, e.g., soil, and the reception of the transmitted or reflected signal. The behaviour of the traveling wave depends on the dielectric properties of the medium itself, according to the EM field theory. In more detail, the wave velocity is influenced by the dielectric permittivity $\varepsilon$, whereas signal loss and attenuation are mainly affected by the electric conductivity $\sigma$. The magnetic permeability $\mu$ is equal to the free space magnetic permeability $\mu_{0}$ for all the nonmagnetic materials, and does not affect the propagation of the EM wave.

In practical terms, when the EM impulse emitted by a source encounters a dielectric contrast in the medium, it generates a partial reflection and transmission of the signal. By collecting such reflections through a receiving antenna, it is possible to image the subsurface features, in both two or three dimensions.

Nowadays, several different GPR systems are available in the market. Main differences are to be related to the type of the antenna and centre frequency of investigation as well as to the working system. A comprehensive presentation of the theoretical and practical features of GPR is given by (Daniels 2004, Jol 2009).

\subsection{Railway applications}

The use of GPR for the investigation of railway substructures has mostly developed over the last 20 years. Originally, GPR surveys were carried out using ground-coupled antennas, with centre frequencies usually below $500 \mathrm{MHz}$. Although these antennas do not provide high resolution, they ensure deep signal penetration. In the last decade, low-frequency antennas have been replaced by higher frequency (1 $\mathrm{GHz} \div 2 \mathrm{GHz}$ ) air-coupled systems, bringing many advantages in terms of ballast degradation assessment (Roberts et al. 2006). Indeed, air-coupled antennas are installed on track inspection vehicles, capable to 
monitor the track conditions continuously and without any interference with rail traffic (Al-Qadi et al. 2008, Fontul et al. 2014). On the other hand, higher frequencies provide greater resolutions and they allow detecting changes in voids volume of ballast, which are likely linked to fouling phenomena (Roberts et al. 2006).

Different features of the GPR signals collected in railway investigations have been taken into account by experts and related to several substructure quality parameters. In (Hugenschmidt 2000), a ground-coupled $900 \mathrm{MHz}$ system mounted onto a cart and towed by a locomotive was employed for surveying a railway section. A comprehensive data post-processing allowed the author assessing the ballast thickness and identifying several zones of clay migration upwards. Olhoeft \& Selig (2002) used an instrumented vehicle equipped with several air-launched antennas with different centre frequencies to perform a visual multifrequency analysis of GPR signals collected on both the centreline and sides of the track. The authors argued that the highest performances were reached with the $1 \mathrm{GHz}$ antenna. They also showed how different results coming from tests performed at the sides of the track may indicate stability issues.

A comparison between data from $1 \mathrm{GHz}$ and 2 $\mathrm{GHz}$ air-coupled antennas was performed by Roberts et al. (2006). It was noticed how the higher frequency was capable to detect differences in the scattering of EM waves, due to the pollution of air voids within the ballast.

More recently, frequency-based studies on railway fouling detection can be found in the literature. In (Al-Qadi et al. 2008), the authors presented a multimethod approach for assessing the ballast condition, based on direct analysis, scattering amplitude envelope, and short time Fourier transform (STFT). A 2 $\mathrm{GHz}$ centre frequency antenna was used for the surveys.

By means of complex experimental and theoretical efforts, Shao et al. (2010) proposed an automatic algorithm for the classification of ballast conditions, based on the application of support vector machine (SVM) onto processed GPR frequency spectra. The method relies on the assumption that the frequency spectrum is affected by the fouling conditions, in a predictable way, which involves the possibility to recognize the ballast quality by analyzing the relevant frequency spectrum.

Fewer laboratory activities can be found in the literature, although they provide essential information for calibrating railway-based models through a proper characterization of the surveyed materials (Shangguan et al. 2012, De Chiara et al. 2014, Fontul et al. 2014, Benedetto et al. 2016a, Tosti et al. 2016).

Also in view of the above studies, a relatively new railway-related research branch began to capture scientists' interest over the last years (Benedetto et al 2016b), namely, the numerical simulation of both physical and dielectric properties of ballasted trackbeds. Indeed, the simulation of the ballasted structure in virtual environment leads to undeniable advantages. One of the most outstanding is the possibility to replicate and extend the experimental conditions, in terms of both frequencies of investigation and track-bed characterization, by properly setting the numerical parameters, which is a massive save of time and economical resources.

\subsection{Advantages and limitations}

Although GPR and NDTs in general often allow to overcome the limits of traditional approaches, a deep knowledge about their limitations is required. This is a simple rule to avoid overestimation of the methods and misinterpretation of the results, which may lead to detrimental effects.

Undeniably, the employment of air-coupled system mounted onto rail vehicles grants a traffic-speed continuous data collection, which involves no disturbance to the railway traffic and a comprehensive monitoring of the track. Furthermore, a regularly scheduled GPR survey is definitely much more costeffective, if compared to traditional tests. It also holds very few concerns about safety issues, which cannot be said for traditional track-bed drilling and trenching.

In turn, GPR is not a direct method, as it measures the potential effects of fouling on dielectric properties of the whole track-bed. Since such effects can be produced by multi-sources, specialist expertise in the interpretation of the data is mandatory, even in the case of automatic methods. Furthermore, as the EM behaviour of the ballast is highly influenced by size distribution and mineralogy of the aggregates, a proper characterization is always required for calibration purposes. In such a framework, numerical simulation can represent a useful mean of integration for GPR.

In general terms, GPR appears as a powerful tool for reducing costs and simultaneously increasing the effectiveness of railway ballast monitoring. Nevertheless, due to its limitations it cannot be employed as a stand-alone technology at the current state of the art, and it grants the highest performance when integrated with other methods.

\section{SCHEME OF EFFICIENT MAINTENANCE}

In this Section, a procedural GPR-based scheme for performing efficient railway maintenance is proposed. Once taken into account the complex environment of railways and the relevant features of GPR, a prompt for an early-stage and non-destructive fouling detection can be listed hereafter (Figure 2). 


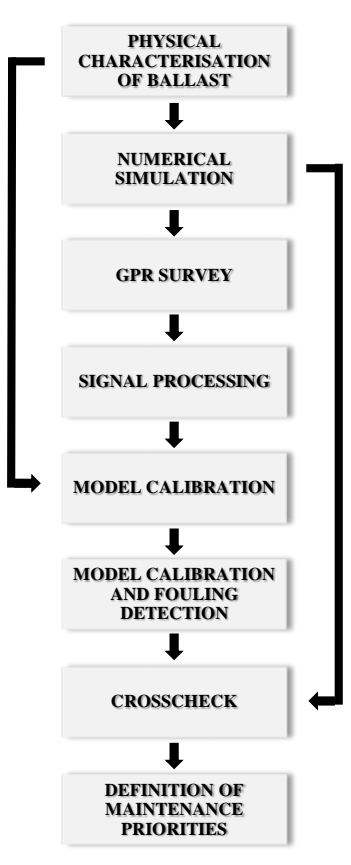

Figure 2. Scheme of effective maintenance using GPR.

1. Physical characterization of ballast. The first step necessarily consists in a comprehensive knowledge of the physical and dielectric properties of the rock-derived aggregates. Such information can be derived from "ad hoc" laboratory tests performed on materials excavated in situ, or it can be provided directly by the railway network administration. In addition, if the mineralogical nature of the ballast is known, shape and size of the aggregates can be assessed by $2 \mathrm{D}$ or $3 \mathrm{D}$ laser scan tests.

2. Numerical simulation. Once the aggregates and the subgrade are characterized, it is possible to reproduce the survey environment within a virtual scenario, numerically simulated. Thereby, synthetic GPR signals related to different fouling conditions can be generated.

3. GPR survey. Hence, GPR data can be collected along the railway section or network. In view of the above, most effective radar configurations are equipped with air-coupled antennas, with centre frequencies ranging between $1 \mathrm{GHz}$ and $2 \mathrm{GHz}$.

4. Signal processing. The collected signal requires now a proper processing in order to remove noise and clutter and, more in general, to reduce the ambiguities and uncertainties. The processing technique must be chosen with reference to the specific model that is planned to be used.

5. Model calibration. The parameters used in the selected model need to be calibrated with regard to the physical properties of the tested material.

6. Model application and fouling detection. The model can now be applied to the database collected along the track. The expected output is an overall assessment of the track-bed, and the identification of the most fouled areas.

7. Crosscheck. The unambiguousness of the results can be significantly raised through a comparison between the data collected on-site and the simulated ones.

8. Definition of the maintenance priorities. If the results are confirmed by simulation, a map of ballast deterioration throughout the whole track is now available, and therefore it is possible to prioritize maintenance actions.

\section{CONCLUSIONS}

This paper aims at reviewing existing practices for the maintenance of railway assets and it introduces a new methodology scheme for the application of effective GPR-based maintenance actions, based on the most recent and up-to-date processing approaches developed in this area of expertise. The first Section introduces the problem, which is tackled more thoroughly in the second Section. Insights on the potential of GPR are given in Section 3, along with a short review of the most acknowledged scientific evidences by literature. In view of the above, several assumptions have been made. Firstly, the most efficient and effective GPR configuration for railway applications is composed of air-coupled pulsed systems, mounted onto rail vehicles and equipped with a high frequency antenna. Secondly, accuracy and reliability of GPR can reach proper standards when integrated with complementary methods. Accordingly, in the maintenance scheme proposed in Section 4, it is worthwhile noting that the application of a GPR model requires proper calibration by a physical characterization of the materials (e.g., performed by laser scanner), as well as a crosscheck by numerical simulation, to validate the results. To that effect, an integration of different methodologies would seem the best solution.

\section{ACKNOWLEDGEMENTS}

This work has benefited from the network activities carried out within the EU funded COST Action TU1208 "Civil Engineering Applications of Ground Penetrating Radar."

\section{REFERENCES}

Al-Qadi, I.L., Xie, W. \& Roberts, R. 2008. Time-frequency approach for ground penetrating radar data analysis to assess railroad ballast condition. In Research in Nondestructive Evaluation, 19, pp. 219-237.

Al-Qadi, I.L., Xie, W., Roberts, R. \& Leng Z. 2010. Data analysis techniques for GPR used for assessing railroad ballast in high radio-frequency environment. In Journal of Transportation Engineering, 136(4), pp. 96-105. 
Benedetto, A., Tosti F., Bianchini Ciampoli L., Calvi A., Brancadoro M.G. \& Alani M.A. 2016a. Electromagnetic characterization of clean and fouled railway ballast through gpr signal processing and numerical simulation. In Construction and Building Materials, In press.

Benedetto, A., Tosti, F., Bianchini Ciampoli, L., Pajewski, L., Pirrone, D., Umiliaco, A. \& Brancadoro, M.G. 2016b. A simulation based approach for railway applications using GPR. In proc. of $16^{\text {th }}$ International Conference on Ground Penetrating Radar (GPR 2016), Hong Kong, June 13-16.

Cho, G.C., Dodds, J. \& Santamaria, J.C. 2006. Particle shape effectson packing density, stiffness and strength of natural and crushed sands. In Journal of Geotechnical and Geoenvironmental Engineering, 132(5), pp. 591-602

Chrismer, S. M. 1985. Considerations of factors affecting ballast performance. In American Railway Engineering Association (AREA), Bulletin 704, AAR Research and Test Department Report No. WP-110, pp. 118-150.

Daniels, D.J. 2004. Ground Penetrating Radar, The Institution of Electrical Engineers, London.

De Chiara, F., Fontul, S. \& Fortunato E. 2014. GPR laboratory tests for railways materials dielectric properties assessment, In Remote Sensing, 6(10), pp. 9712-9728.

Ebrahimi, A., Tinjum, J.M. \& Edil T.B. 2012. protocol for testing fouled railway ballast in large-scale cyclic triaxial equipment, In Geotechnical Testing Journal, 35 (5), pp. 1-9.

Fontul, S., Fortunato, E. \& De Chiara F. 2014. evaluation of ballast fouling using GPR, In proc. of $15^{\text {th }}$ International Conference on Ground Penetrating Radar (GPR 2014), Brussels, June 30 - July 4.

Hyslip, J.P., Smith, S.S., Olhoeft, G.R. \& Selig E.T. 2003. Assessment of railway track substructure condition using ground penetrating radar, in Proc. Annu. Conf. AREMA, Chicago, NY.

Huang, H., Tutumluer, E., Hashash, Y., \& Ghaboussi, J. 2010. Laboratory validation of coal dust fouled ballast discrete element model, In Paving Materials and Pavement Analysis, pp. 305-313.

Hugenschmidth, J. 2000. Railway track inspection using GPR, In Journal of Applied Geophysics, 43, pp. 147-155
Indraratna, B. 2016). 1st Ralph Proctor lecture of ISSMGE. Railroad performance with special reference to ballast and substructure characteristics, In Transportation Geotechnics, 7, pp. 74-114.

Indraratna, B., Nimbalkar, S., Coop, M. \& Sloan S.W. 2014. A Constitutive model for coal-fouled ballast capturing the effects of particle degradation, In Computer and Geotechnics, 61, pp. 96-107.

Jol, H. 2009. Ground penetrating radar: theory and applications, In Elsevier ed., Amsterdam, The Netherlands.

Olhoeft, G.R. \& Selig, E.T. 2002. Ground penetrating radar evaluation of railway track substructure conditions, In $9^{\text {th }}$ International Conference on Ground Penetrating Radar (GPR 2002), Santa Barbara, California, April 29 - 2 May.

Roberts R., Rudy J., Al-Qadi I.L., Tutumluer E. \& Boyle J. 2006 Railroad ballast fouling detection using ground penetrating radar - a new approach based on scattering from voids, In $9^{\text {th }}$ European Conference on NDT.

Selig E. T. \& Waters J. M. 1994. Track geotechnology and substructure management. In Thomas Telford ed., London.

Shangguan, P., Al-Qadi, I.L. \& Leng Z. 2012. Ground-penetrating radar data to develop wavelet technique for quantifying railroad ballast-fouling conditions, In Transportation Research Record, 2289, pp. 95-102.6

Shao, W., Bouzerdoum, A., Phung, S.L., Su, L., Indratatna, B. Rujikiatkamjorn C. 2010. Automatic classification of ground-penetrating-radar signals for railway-ballast assessment, In IEEE Transactions on Geoscience and Remote Sensing Vol. 49(10), pp. 3961-3972.

Sun, Y., Indraratna, B. \& Nimbalkar S. 2014. Three-dimensional characterisation of particle size and shape for ballast, In Géotechnique Letters, 4 (3), pp. 197-202.

Tennakoon, N., Indraratna, B., Nimbalkar, S. \& Sloan S.W. 2015. Application of bounding surface plasticity concept for clay-fouled ballast under drained loading, In Computer and Geotechnics, 70, pp. 96-105.

Tosti, F., Benedetto, A., Calvi, A., \& Bianchini Ciampoli, L. 2016. Laboratory investigations for the electromagnetic characterization of railway ballast through GPR. In Proc. 16th International Conference of Ground Penetrating Radar (GPR 2016), Hong Kong, June 13-16, 2016. 